\title{
High-Resolution Electron Beam Lithography and DNA Nano-Patterning for Molecular QCA
}

\author{
Wenchuang Hu, Member, IEEE, Koshala Sarveswaran, Marya Lieberman, and Gary H. Bernstein, Senior Member, IEEE
}

\begin{abstract}
Electron beam lithography (EBL) patterning of poly(methylmethacrylate) (PMMA) is a versatile tool for defining molecular structures on the sub-10-nm scale. We demonstrate lithographic resolution to about $5 \mathrm{~nm}$ using a cold-development technique. Liftoff of sub-10-nm Au nanoparticles and metal lines proves that cold development completely clears the PMMA residue on the exposed areas. Molecular liftoff is performed to pattern DNA rafts with high fidelity at linewidths of about 100 nm. High-resolution EBL and molecular liftoff can be applied to pattern Creutz-Taube molecules on the scale of a few nanometers for quantum-dot cellular automata.
\end{abstract}

Index Terms-Au nanoparticles, DNA tiling, electron beam lithography (EBL), liftoff, metal grains, molecular electronics, nanofabrication, quantum-dot cellular automata (QCA).

\section{INTRODUCTION}

$\mathbf{T}$ HE International Technology Roadmap for Semiconductors predicts integrated circuits with gate lengths of $9 \mathrm{~nm}$ by the year 2016 [1]. Given the amazing progress by industry, including the tendency to perform even better than forecasts, as well as recent results of gate lengths approaching $10 \mathrm{~nm}$ [2], it is reasonable to assume that future predictions will be realized. ${ }^{1}$ Nevertheless, issues of overhead on total transistor area and power dissipation leave some room for continued evolution and miniaturization, although not necessarily in the Si domain. The ultimate size scale for future computing devices lies in the domain of molecular electronics. However, the conventional paradigm of gating molecules seems impractical. Recognizing that the whole field of molecular electronics is in a nascent phase, one of the most promising uses of molecules for computing is that of quantum-dot cellular automata (QCA) [3]-[5]. In this paradigm, molecules are capacitively clocked and field-coupled to neighbors, so that most individual contacts are not necessary. With QCA, it is predicted that high speed and low power can be achieved.

In general, "top-down" lithographic methods have difficulties in satisfying the requirements of both resolution and throughput. On the other hand, self-assembly has shown the capability for patterning nanocomponents, such as nanoparticles

\footnotetext{
Manuscript received June 11, 2004; revised December 1, 2004. This work was supported in part by the Office of Naval Research and the Defense Advanced Research Projects Agency under Grant N00014-01-1-0658, in part by Intel Corporation, in part by the W. M. Keck Foundation, and in part by the National Science Foundation under Grant ECS-03-04630.

W. Hu and G. H. Bernstein are with the Department of Electrical Engineering, University of Notre Dame, Notre Dame, IN 46556 USA (e-mail: gbernste@nd.edu).

K. Sarveswaran and M. Lieberman are with the Department of Chemistry and Biochemistry, University of Notre Dame, Notre Dame, IN 46556 USA.

Digital Object Identifier 10.1109/TNANO.2005.847034
}

${ }^{1}$ See also http://www.eetimes.com/semi/news/OEG20020909S0106. and nanocrystals on the molecular scale [6]-[9] and creating an extraordinary level of structural complexity [9]. However, the manufacturing of bottom-up assembled systems with such stereochemical complexity would be extremely difficult. A possible solution to this manufacturing challenge is an appropriate combination of top-down lithography with self-assembly.

The ultimate goal here is to construct molecular quantum-dot cellular automata (MQCA) by allowing molecules or DNA (deoxyribonucleic acid) tiles as QCA cells to self-assemble on templates formed by electron beam lithography (EBL) involving one step of removal of poly(methylmethacrylate) (PMMA) by liftoff. This technique has proven viable for both Creutz-Taube molecules [10] and larger DNA nanostructures. This work on DNA patterning is a preliminary study of the combination of lithography and self-assembly. We have begun the investigation of using self-assembly to build functional molecular sub-systems on DNA rafts and EBL and molecular liftoff to position the rafts into macroscopic architectures.

Fabrication of molecular electronics and particularly QCA requires high-resolution methods to pattern molecules with a few nanometer in size. EBL is still the most versatile of the high-resolution patterning techniques, but is not generally capable of writing below the $10 \mathrm{~nm}$ required of molecular structures. Since even large molecules do not approach this size, it is important to be able to define patterns well below $10 \mathrm{~nm}$. Here we report a cold-development technique used to break the 10-nm barrier with good control over patterning properties, to about $5 \mathrm{~nm}$. Based on our previous work [11], we further proved the thorough development of our resist patterns using cold developer and metal liftoff along with Au nanoparticle liftoff. At this scale, perhaps a few, or even one large molecule can fit laterally within a PMMA trench. Based on the chosen chemistry, self-assembly of the molecules within the trenches will guarantee coherent pattern formation suitable for MQCA.

\section{5-nm EBL With Cold DEVELOPMENT}

EBL is performed using our $30-\mathrm{kV}$ cold-cathode field emission Hitachi S-4500 scanning electron microscope converted to EBL [12]. The spot size is about $1 \mathrm{~nm}$. One advantage of using this system is the ease of achieving optimum beam parameters prior to exposure, assuring that the best possible lithography results can be reliably achieved. We have used this system to investigate the effects of varying the developer temperature on ultimate lithographic resolution, and found that cooling the developer yields noticeable advantages.

For EBL, 30-70 nm, 950 K amu PMMA was used as a positive resist. Isopropyl alcohol: methyl isobutyl ketone $(3: 1)$ with $1.5 \mathrm{vol} \%$ methyl ethyl ketone was used as developer because of its high contrast [13]. During development, $10 \mathrm{ml}$ of developer 


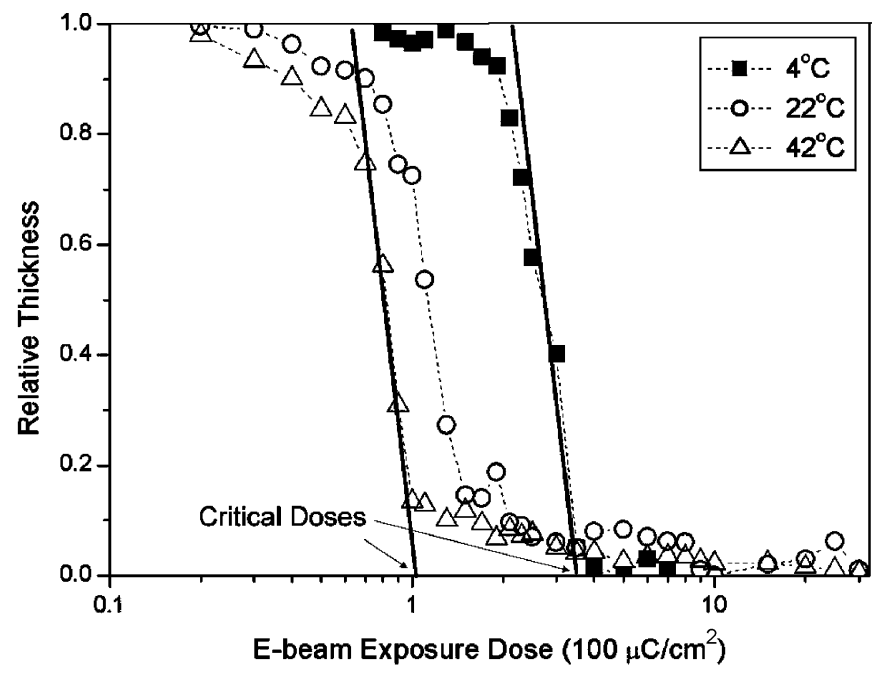

Fig. 1. PMMA contrast curves at development temperatures of $4{ }^{\circ} \mathrm{C}, 22^{\circ} \mathrm{C}$, and $42{ }^{\circ} \mathrm{C}$, measured by AFM metrology.

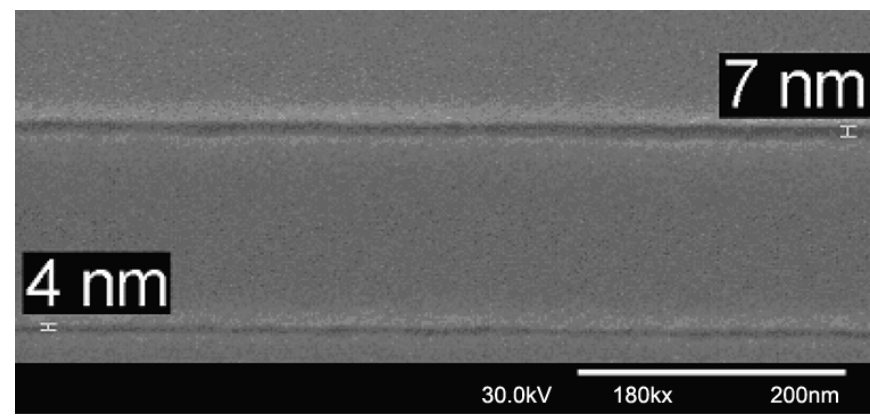

Fig. 2. 4-7-nm PMMA trenches sputter-coated with 2-nm $\mathrm{Cr}$ developed by cold development.

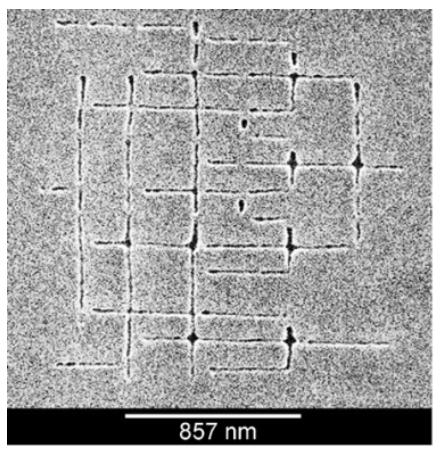

a

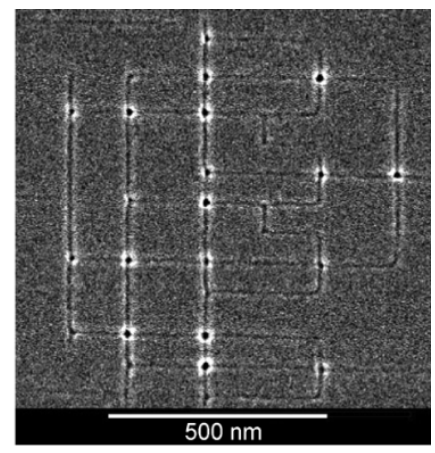

b
Fig. 3. QCA adder patterns in PMMA resists developed at (a) $23{ }^{\circ} \mathrm{C}$ and (b) $6^{\circ} \mathrm{C}$.

was cooled to the desired temperature of $4{ }^{\circ} \mathrm{C}$ to $8{ }^{\circ} \mathrm{C}$ with a precision of $1{ }^{\circ} \mathrm{C}$. Development time varied from 30-90 s depending on the resist thickness $(30-80 \mathrm{~nm})$ and developer temperature $\left(4^{\circ} \mathrm{C}\right.$ to $\left.8^{\circ} \mathrm{C}\right)$. Resist patterns were coated with 1-2-nm $\mathrm{Cr}$ or $\mathrm{Au}_{60} \mathrm{Pd}_{40}$ by an Emitech model 660 plasma sputter coater or a Varian thermal evaporator for SEM examination.

Contrast curves at three different temperatures were measured by atomic force microscopy (AFM) metrology [11], as shown in Fig. 1. The significant feature is that for cold development the tail usually present in these curves is closer to the

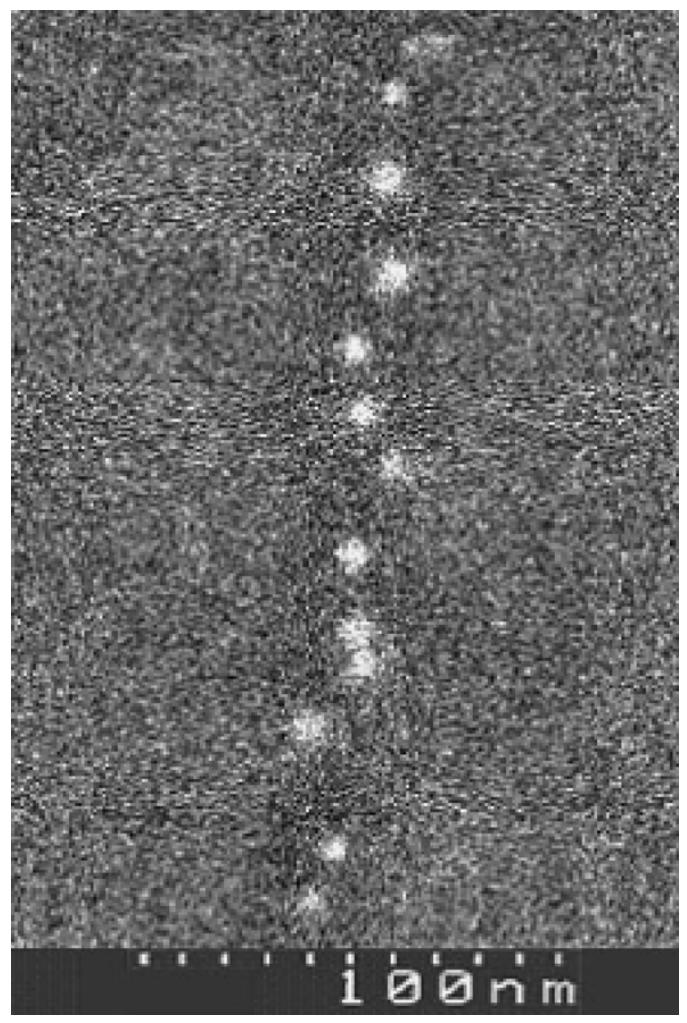

Fig. 4. Lifted-off line with one 5-nm Au nanoparticle in the width. The flow of nanoparticles to the edges of trenches caused the spreading of line.

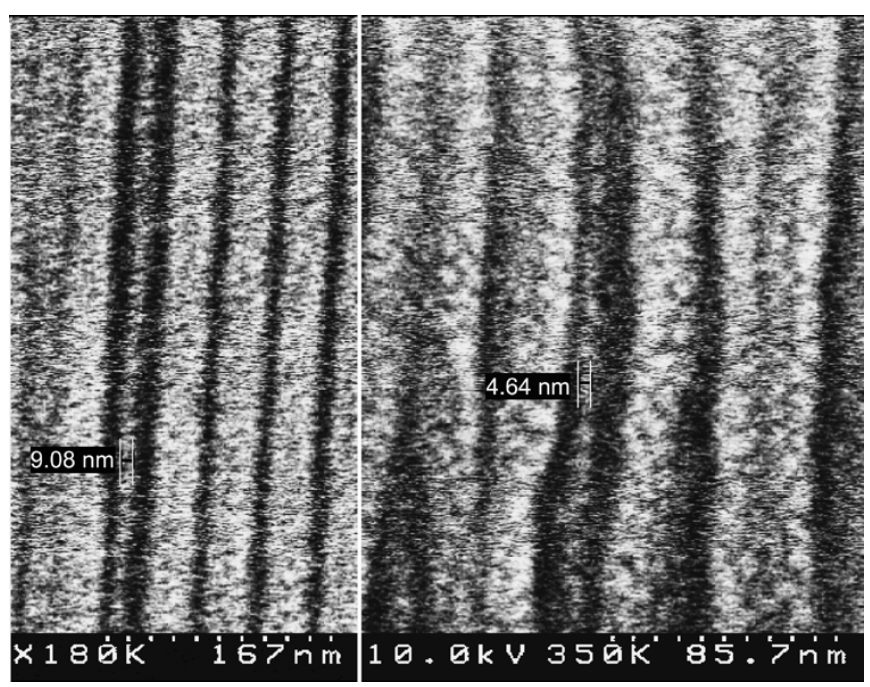

Fig. 5. $\mathrm{Au}_{60} \mathrm{Pd}_{40}$ lines ( $3 \mathrm{~nm}$ thick) with 5-10-nm width formed in 40-nm-deep PMMA trenches after PMMA removal. Formation of 5-nm lines proves complete development of narrow PMMA trenches by the cold-development technique.

critical dose (where the straight portion of the curve extrapolates to zero), so less overdose is necessary to ensure clearing of the patterns, and higher resolution results. Fig. 2 shows resist developed by cold developer and coated with 2-nm Cr. The linewidths approach and perhaps surpass 5-nm width for 40-nm thick PMMA.

The key problem for sub-10-nm EBL is the incomplete development of exposed resist patterns and sometimes the appearance 
$\mathbf{A}$

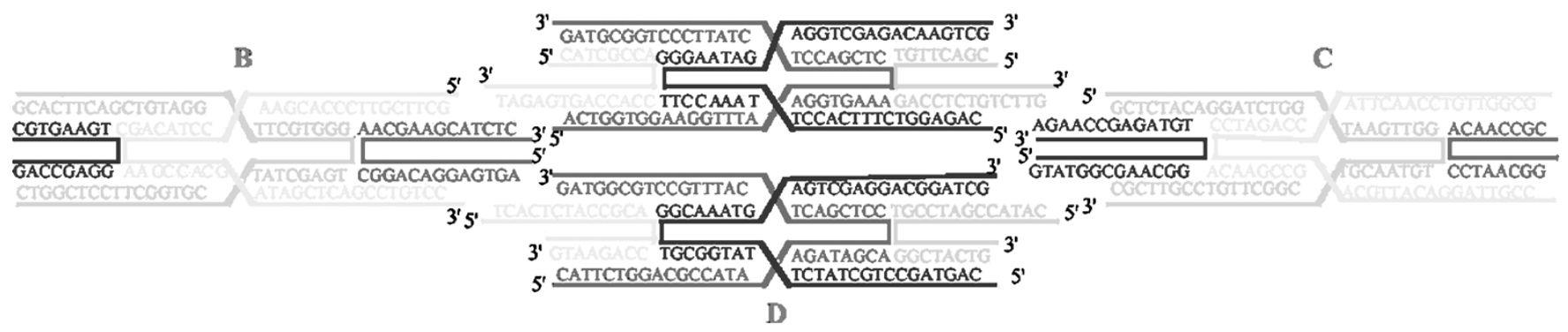

Fig. 6. Schematic structure of four-tile DNA raft [23].

of bridging effects [14]. We found cold development can eliminate this problem and therefore indicates complete development of sub-10-nm resist patterns. Fig. 3 compares two QCA adder patterns in PMMA. Fig. 3(a) is the sample developed at room temperature $\left(23^{\circ} \mathrm{C}\right)$ with a line dose of $3.5 \times 10^{-10} \mathrm{C} / \mathrm{cm}$ and Fig. 3(b) is for cold development $\left(6^{\circ} \mathrm{C}\right)$ with a line dose of $8.5 \times$ $10^{-10} \mathrm{C} / \mathrm{cm}$. The linewidth in each pattern is $6-10 \mathrm{~nm}$, and the PMMA thickness and developer were the same. The room temperature sample shows obvious bridging in the lines while the cold-development sample has no such effect, which indicates cold development offers complete development of sub-10-nm PMMA patterns.

Liftoff is nearly the only way to transfer high resolution of EBL from PMMA due to its poor etch resistance. For sub-10-nm liftoff, the most important issue is that the bottom of the narrow trenches must be clean of resist residue. To test the existence of resist residue in the trenches after cold development, we deposited 5-nm negatively charged gold nanoparticles [15] into the positively charged trenches (trench bottom was coated with polylysine after development). PMMA was then removed by dichloromethane $\left(\mathrm{CH}_{2} \mathrm{Cl}_{2}\right)$ [16]. As can be seen in Fig. 4, a line of single particles is obtained, which would not be possible without a clean oxide surface at the bottom of the trench since the particles do not stick to PMMA residue. This result also demonstrates an example application of EBL to pattern sub-10-nm structures. These nanoparticle structures can be used for single electron devices [17], [18].

Besides Au nanoparticle liftoff, we deposited and lifted off granular metal structures [19]. After EBL and cold development, 2-3 nm thick $\mathrm{Au}_{60} \mathrm{Pd}_{40}$ was deposited by thermal evaporation. Then liftoff was performed in warm acetone for about $30 \mathrm{~min}$. AuPd lines with 5-10-nm widths were well formed in the PMMA trenches, as shown in Fig. 5. In these cases, we did not use ultrasonic agitation or syringe blowing during liftoff due to the weak adhesion of these thin metal lines to the silicon substrate. PMMA thickness was $30-40 \mathrm{~nm}$. However, the top metal layer stuck to the substrate after PMMA removal. The combination of thin resist and thin metal film would likely be the reason to cause this problem. The bulk silicon substrates were charged by EBL and could strongly attract the thin metal films by electrostatic forces when the thin resist was removed during liftoff. The thinner the resist, the less able is the metal to stand up to. However, the key point here is that the formation of the 5-nm-wide metal line proves that the PMMA trenches were completely developed from the top to the substrate surface on the 5-nm scale. This conclusion is critical for the molecular liftoff of QCA cells.
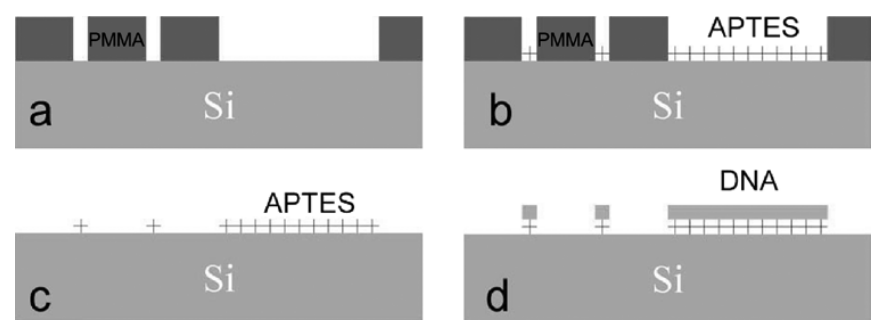

Fig. 7. Molecular liftoff of APTES for DNA attachment.

PMMA masks defined by EBL can be used to perform a molecular lift-off technique [10] wherein hydrophilic molecules preferentially stick to a $\mathrm{SiO}_{2}$ surface. A 15 -nm-wide nanoline of Creutz-Taube ion $\left[\left(\mathrm{NH}_{3}\right)_{5} \mathrm{Ru}(\right.$ pyrazine $\left.)-\mathrm{Ru}\left(\mathrm{NH}_{3}\right)_{5}\right](o-$ toluenesulphonate $)_{5}$ (CT5) [20] molecules has been demonstrated [21]. For future work, the fabrication of sub-10-nm single-molecule nanolines is very possible given the completely developed narrow trenches by the cold-development technique.

\section{NANOPATTERNING OF DNA RAFTS}

As another approach to fabricate molecular QCA, we aim to create a self-assembled DNA lattice with maximally controlled heterogeneity for QCA cells [22]. Four-tile DNA rafts have been successfully synthesized and were characterized by the gel electrophoresis method in our previous work [22], [23]. Fig. 6 shows the structure of the four-tile raft, which measures about $8 \times 37 \times 2 \mathrm{~nm}^{3}$. At first, we used the liftoff process as Au nanoparticle. However, polylysine as an adhesion layer for DNA attachment turned out to be unsuitable due to denaturation of the polylysine and DNA rafts by the methylene chloride used for liftoff (results not shown). In order to effect controlled deposition of DNA rafts without such damage, we revised the liftoff process, as illustrated in Fig. 7. A SAM of APTES (3-Aminopropyl-triethoxysilane) was deposited in the trench bottoms from aqueous solution. The PMMA resist was dissolved in $\mathrm{CH}_{2} \mathrm{Cl}_{2}$ for $3 \mathrm{~min}$, and the surface was rinsed with water. Next, $20 \mu \mathrm{l}$ of DNA raft solution $(1 \mu \mathrm{M})$ was deposited on the surface and left for about $4 \mathrm{~h}$. After the DNA rafts selectively attached to the APTES patterns, the surface was rinsed with water, dried with nitrogen, and then imaged in AFM. Using this method, we were able to pattern DNA rafts with high fidelity. Fig. 8 shows the lifted off APTES lines and the attached DNA rafts on these lines.

The roughness of the substrate, the PMMA trench bottom after development, and the substrate after stripping of PMMA 

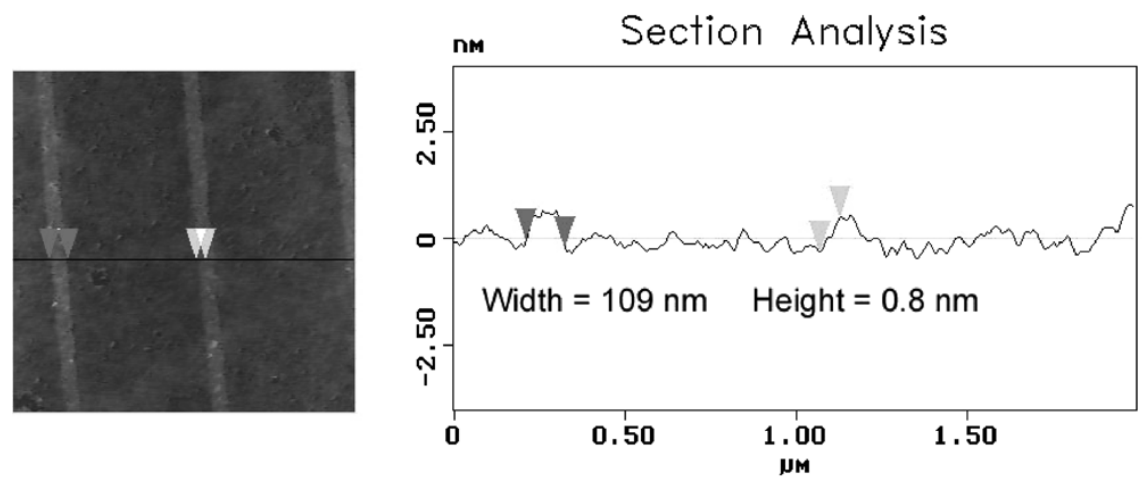

a
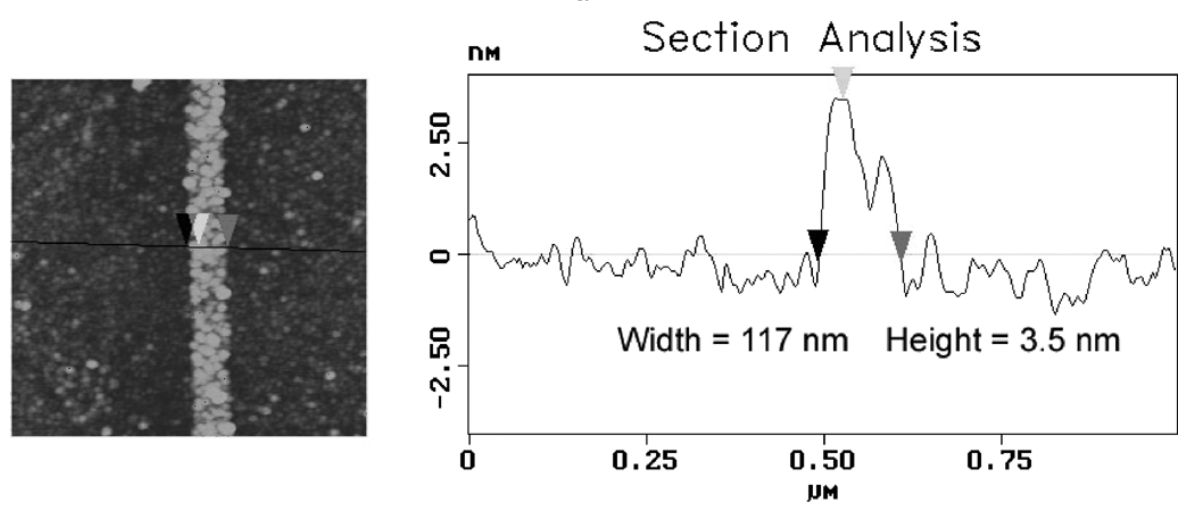

b

Fig. 8. AFM images and profiles of (a) APTES lines by liftoff and (b) DNA rafts attached to the APTES lines. A low level of nonspecific attachment of the DNA rafts to the silicon oxide substrate is observed due to the mutual repulsion of the anionic oxide and the anionic DNA backbone.

are important for the liftoff process. An improved cleaning procedure [24] produced extremely smooth substrate surfaces, about $0.05 \mathrm{~nm}$. The silicon roughness in the PMMA trenches after development was about $0.3 \mathrm{~nm}$, and that of the silicon surface after PMMA removal by dichloromethane [16] instead of acetone is about $0.25 \mathrm{~nm}$.

Control experiments along with X-ray photoelectron spectroscopy measurements [25] show that APTES offers excellent adhesion and coverage uniformity on smooth $\mathrm{SiO}_{2}$. Also, keeping APTES-soaked samples in a vacuum desicator overnight removes the loosely bound APTES on the PMMA and prevents the possible extra attachment of APTES on silicon during the PMMA removal step. As shown in Fig. 8, the height of the APTES monolayer measures about $0.8 \mathrm{~nm}$ and the height of the DNA lines on APTES measures about $2.7 \mathrm{~nm}$, which is a good match for that of the DNA rafts ( $2 \mathrm{~nm}$ for duplex DNA) plus the height of the APTES $(0.8 \mathrm{~nm})$ monolayer.

\section{CONCLUSION}

In summary, we have demonstrated ultrahigh resolution (about $5 \mathrm{~nm}$ ) EBL in PMMA resist by the use of a cold-development technique and the cold cathode field emission system. The liftoff process is a feasible method to transfer such ultrahigh resolution to various nanofabrication applications. The key issue for sub-10-nm liftoff is the complete development of resist residue from the sub-10-nm high aspect-ratio trenches. Our results of Au nanoparticle and metal liftoff prove that cold development satisfies such a key requirement. 5-nm EBL resolution has been successfully transferred to metal and nanoparticle structures.
EBL with molecular liftoff is a promising approach to pattern molecules and DNA rafts for the fabrication of MQCA, and molecular electronics in general. Liftoff of CT5 molecules in a controlled manner has been achieved with resolution to about $15 \mathrm{~nm}$. The use of our cold-development technique will likely enable us to fabricate single-molecule nanolines. In order to image these narrow lines and investigate surface properties, scanning tunneling microscopy (STM) is proposed to be used for the atomic resolution.

A well-controlled molecular liftoff process was developed to pattern four-tile DNA rafts onto silicon substrates with low level defects and low surface roughness. 100-nm-wide APTES and DNA lines were fabricated. However, there are clearly some issues that need to be addressed. For instance, a potential problem is the apparent irreversibility of the binding interaction, which may interfere with attempts to create short range ordering through raft-raft interactions. Another problem is how to orient DNA rafts in the EBL defined patterns. Future work in the DNA liftoff will focus on the possibility of DNA rafts as structural templates for non-DNA materials attachment, the scalability of the raft size from 4 to 54 tiles, and the orientation of DNA rafts in the EBL patterns.

It is not likely that a completely self-assembled circuit of sufficient complexity can be achieved, but some self-assembly would be used to connect molecules together within a pattern. Such patterns would likely be on the order of one to a few molecules in width. Furthermore, it is likely that a large fraction of a conventional CMOS chip would be patterned with molecules to interface with conventional CMOS. Using EBL with a sufficiently small beam, even with the brightest source, it would take several hours to pattern a single chip, but tech- 
niques such as nanoimprint lithography may serve as an intermediate step from the EBL techniques demonstrated here and future manufacturing technologies.

\section{REFERENCES}

[1] Semiconductor Industry Assoc. et al., "International Technology Roadmap for Semiconductors 2002 update," SIA, San Jose, CA, 2002. [Online]. Available: http://public.itrs.net/Files/2002Update/2002Update.pdf.

[2] B. Doyle, R. Arghavani, D. Barlage, S. Datta, M. Doczy, J. Kavalieros, A. Murthy, and R. Chau, "Transistor elements for $30 \mathrm{~nm}$ physical gate lengths and beyond," Intel Technol. J., vol. 6, no. 2, May. [Online]: Available: http://www.intel.com/technology/itj/2002/volume06issue02/art05_transistorarch/p01_abstract.htm.

[3] C. S. Lent, P. D. Tougaw, W. Porod, and G. H. Bernstein, "Quantum cellular automata," Nanotechnology, vol. 4, p. 49, 1993.

[4] M. Lieberman, S. Chellamma, B. Varughese, Y. Wang, C. Lent, G. H. Bernstein, G. Snider, and F. C. Peiris, "Quantum-dot cellular automata at a molecular scale," in Molecular Electronics II 2002 Annals of the New York Academy of Sciences. New York: New York Acad. Sci., vol. 960, pp. 225-239.

[5] C. S. Lent, B. Isaksen, and M. Lieberman, "Molecular quantum-dot cellular automata," J. Amer. Chem. Soc., vol. 125, no. 4, pp. 1056-1063, 2003.

[6] M. Li, H. Schnablegger, and S. Mann, "Coupled synthesis and self-assembly of nanoparticles to give structures with controlled organization," Nature, vol. 402, pp. 393-395, 1999

[7] C. J. Loweth, W. B. Caldwell, X. G. Peng, A. P. Alivisatos, and P. G. Schultz, "DNA-based assembly of gold nanocrystals," Angew. Chem. Int. Ed. Engl., vol. 38, pp. 1808-1812, 1999.

[8] D. J. Norris and Y. A. Vlasov, "Chemical approaches to three-dimensional semiconductor photonic crystals," Adv. Mater, vol. 13, pp. $371-376,2001$

[9] J. C. Ellenbogen and J. C. Love. (1999) Architectures for molecular electronic computers. 1. Logic structures and an adder built from molecular electronic diodes. MITRE Res. Paper [Online]. Available: http://www.mitre.org/tech/nanotech/downloads/Arch_for_MolecElec_ Comp_1.pdf

[10] Q. Hang, Y. Wang, M. Lieberman, and G. H. Bernstein, "Molecular patterning through high-resolution polymethylmethacrylate masks," Appl. Phys. Lett., vol. 80, no. 22, pp. 4220-4222, 2002.

[11] W. Hu, G. H. Bernstein, K. Sarveswaran, and M. Lieberman, "Sub-10 $\mathrm{nm}$ electron beam lithography using cold development of PMMA," $J$. Vac. Sci. Technol. B, vol. 22, no. 4, pp. 1711-1716, 2004.

[12] W. Hu, T. Orlov, and G. H. Bernstein, "Technique for preparation of precise wafer cross-sections and applications to EBL of PMMA resist,' J. Vac. Sci. Technol. B, vol. 20, no. 6, pp. 3085-3088, 2002.

[13] G. H. Bernstein, D. A. Hill, and W. P. Liu, "New high-contrast developers for PMMA resist," J. Appl. Phys., vol. 71, no. 8, pp. 4066-4075, 1992.

[14] J. M. Ryan, A. C. F. Hoole, and A. N. Broers, "A study of the effect of ultrasonic agitation during development of poly(methylmethacrylate) for ultrahigh resolution electron-beam lithography," J. Vac. Sci. Technol. $B$, vol. 13, no. 6, pp. 3035-3039, 1995.

[15] C. J. Loweth, W. B. Caldwell, X. Peng, A. P. Alivistos, and P. G. Schultz, Angew. Chem. Int. Ed., vol. 38, p. 1808, 1999.

[16] Q. Hang, D. A. Hill, and G. H. Bernstein, "Efficient removers for polymethylmethacrylate," J. Vac. Sci. Technol. B, vol. 21, pp. 91-97, 2003.

[17] T. Sato, D. G. Hasko, and H. Ahmed, "Nanoscale colloidal particles: Monolayer organization and patterning," J. Vac. Sci. Technol. B, vol. 15, no. 1, pp. 45-48, 1997

[18] C. Vieu, F. Carcenac, A. Pepin, Y. Chen, M. Mejias, A. Lebib, L. ManinFerlazzo, L. Couraud, and H. Launois, "Electron beam lithography: Resolution limits and applications," Appl. Surf. Sci., vol. 164, pp. 111-117, 2000

[19] C. Vieu, M. Mejias, F. Carcenac, G. Faini, and H. Launois, "Sub-10 nm monogranular metallic lines formed by $200 \mathrm{kV}$ electron-beam lithography and lift-off in polymethylmethacrylate resist," Microelectron. Eng., vol. 35, no. 1-4, pp. 253-256, 1997.

[20] C. Creutz and H. Taube, J. Amer. Chem. Soc., vol. 95, p. 1086, 1973.

[21] G. H. Bernstein, Q. Hang, W. Hu, K. Sarveswaran, and M. Lieberman, "Nanolithography for molecular electronics," presented at the IEEE Conf. Nanoscale Devices and System Integration, Miami, FL, Feb. $15-19,2004$.

[22] K. Sarveswaran, W. Hu, P. Huber, G. H. Bernstein, M. Niemier, and M. Lieberman, "Self-assembly and lithographic patterning of DNA rafts," presented at the DARPA Conf. Foundations of Nanoscience: Self-Assembled Architectures and Devices, Snowbird, UT, Apr. 2004.
[23] K Sarveswaran, P. Huber, M. Lieberman, C. Russo, and C. Lent, "Nanometer scale rafts built from DNA tiles," in Proc. 3rd IEEE Conf. Nanotechnology, vol. 2, 2003, pp. 417-420.

[24] X. Wang, W. Hu, R. Ramasubramaniam, G. H. Bernstein, G. Snider, and M. Lieberman, "Formation, characterization, and sub-50-nm patterning of organosilane monolayers with embedded disulfide bonds: An engineered self-assembled monolayer resist for electron-beam lithography,' Langmuir, vol. 19, no. 23, pp. 9748-9758, 2003.

[25] K. Sarveswaran, W. Hu, P. Huber, G. H. Bernstein, M. Niemier, and M. Lieberman, Nature materials. Manuscript in preparation.

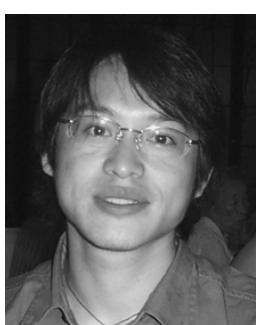

Wenchuang Hu (M'04) received the B.S.E.E. degree from Peking University, Beijing, China, in 1999, and the M.S.E.E. and Ph.D. degrees from the University of Notre Dame, Notre Dame, IN, in 2001 and 2004, respectively.

He is currently working as a Postdoctoral Research Fellow at the Solid State Electronics Laboratory, Department of Electrical Engineering and Computer Science, University of Michigan, Ann Arbor. His research interests are nanofabrication technology and applications, which include electron beam lithography, nanoimprint lithography, nanoelectronics, molecular electronics, biomedical nanodevices, and systems.

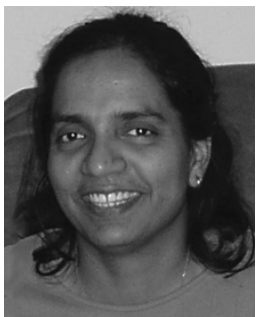

Koshala Sarveswaran received the B.S. degree in chemistry from the University of Jaffna, Sri Lanka, in 1992, and the Ph.D. degree in inorganic chemistry from the University of Cambridge, Cambridge, U.K., in 1999.

In 2000, she became a Post Doctoral Research Associate with the Department of Chemistry and Biochemistry at the University of Notre Dame, Notre Dame, IN. Her current research interests include synthesis and characterization of DNA tiles and investigation of their surface attachment properties. She is also working on the synthesis and characterization of electroactive SAMs.

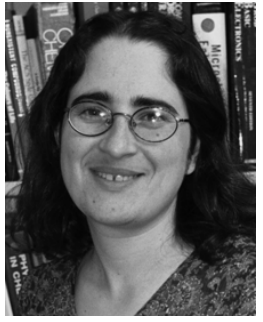

Marya Lieberman studied de novo protein design with Tomi Sasaki at the University of Washington, Seattle, and received the Ph.D. degree in 1994.

She worked on surface chemistry with Nathan Lewis at the California Institute of Technology, Pasadena, as an NSF Postdoctoral Fellow (1994-1996), and joined the Department of Chemistry and Biochemistry, University of Notre Dame in 1996. Her research interests include molecular implementation of quantum-dot cellular automata, unusual self-assembled molecules, ultra-high resolution lithography, and interactions of biomolecules with surfaces.

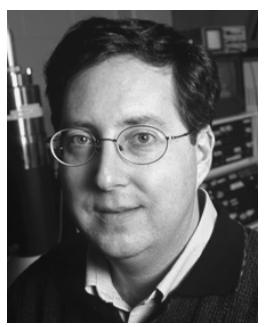

Gary H. Bernstein (SM'95) received the B.S.E.E degree (with honors) from the University of Connecticut, Storrs, in 1979, the M.S.E.E. degree from Purdue University, West Lafayette, IN, in 1981, and the Ph.D. degree from Arizona State University (ASU), Tempe, in 1987.

He then spent a year as a Post-Doctoral Fellow with ASU. In 1988, he joined the Department of Electrical Engineering, University of Notre Dame, Notre Dame, IN, and served as the founding Director of the Nanoelectronics Laboratory from 1989 to 1998 . He is currently Professor and Associate Chair of the Department of Electrical Engineering, University of Notre Dame. He has authored or coauthored over 100 publications in the areas of electron beam lithography, quantum electronics, ultrahigh-speed integrated circuits, reliability of ULSI components, and microelectromechanical systems.

Dr. Bernstein was the recipient of a 1992 National Science Foundation White House Presidential Faculty Fellow Award and a 2001 Notre Dame Kaneb Teaching Award. 